\title{
Predicting MGMT Methylation Status of Glioblastomas from MRI Texture
}

\author{
Ilya Levner ${ }^{1,2,3}$, Sylvia Drabycz ${ }^{4}$, Gloria Roldan ${ }^{5,6,7,8}$, Paula De Robles ${ }^{5,6,7,8}$, \\ J. Gregory Cairncross ${ }^{5,6,7,8}$, and Ross Mitchell ${ }^{1,2,3,6,7,8}$ \\ 1 Department of Radiology, University of Calgary, Alberta, Canada \\ 2 Southern Alberta Cancer Research Institute, University of Calgary, \\ Alberta, Canada \\ 3 Alberta Ingenuity Center for Machine Learning, Canada \\ 4 Department of Electrical and Computer Engineering, University of Calgary, \\ Alberta, Canada \\ 5 Department of Oncology, Tom Baker Cancer Centre, Alberta Cancer Board, \\ Canada \\ ${ }^{6}$ Hotchkiss Brain Institute, Calgary, Alberta, Canada \\ 7 Department of Clinical Neurosciences, University of Calgary, Alberta Canada \\ 8 Clark Smith Brain Tumor Centre, Southern Alberta Cancer Research Institute, \\ Canada
}

\begin{abstract}
In glioblastoma (GBM), promoter methylation of the DNA repair gene MGMT is associated with benefit from chemotherapy. Because MGMT promoter methylation status can not be determined in all cases, a surrogate for the methylation status would be a useful clinical tool. Correlation between methylation status and magnetic resonance imaging features has been reported suggesting that non-invasive MGMT promoter methylation status detection is possible. In this work, a retrospective analysis of T2, FLAIR and T1-post contrast MR images in patients with newly diagnosed GBM is performed using L1-regularized neural networks. Tumor texture, assessed quantitatively was utilized for predicting the MGMT promoter methylation status of a GBM in 59 patients. The texture features were extracted using a space-frequency texture analysis based on the S-transform and utilized by a neural network to predict the methylation status of a GBM. Blinded classification of MGMT promoter methylation status reached an average accuracy of $\mathbf{8 7 . 7 \%}$, indicating that the proposed technique is accurate enough for clinical use.
\end{abstract}

\section{Introduction}

Glioblastoma multiforme (GBM) is the most common primary brain tumor in adults. Standard treatment now includes a DNA alkylating agent, Temozolomide (TMZ), which is the only known chemotherapeutic that prolongs survival 1. Interestingly, the effectiveness of TMZ may be predictable; via a test for methylation of the O6-methylguanine-DNA methyltransferase (MGMT) gene 
promoter. Methylation of the MGMT promoter inhibits the repair of therapeutic DNA damage induced by TMZ thus rendering a drug-resistant cancer more sensitive to chemotherapy [2]. For unknown reasons, MGMT is silenced in $50 \%$ of newly diagnosed GBMs [3]. Therefore, a sensitive and specific test that reliably predicts the methylation status of a given GBM would be a helpful diagnostic alternative to the standard physical biopsy currently employed to diagnose the MGMT status of glioblastomas.

Recently Eoli et al., in 4, found significant correlations between MGMT promoter methylation status and magnetic resonance (MR) imaging features. Motivated by these findings, this research presents an automated method for predicting the methylation status of the GBM based on texture analysis of T2, FLAIR and T1-postcontrast MRI scans. In order to analyze the MR images, the proposed system utilizes the 2-dimensional discrete orthogonal S-transform [5] to extract texture features that are subsequently used by an $\ell_{1}$-Regularized neural network to predict the methylation status of a given GBM. In a leave-one-out cross validation study, the proposed system achieved an average accuracy of $87.7 \%$, high enough for use in clinical diagnosis.

\section{$1.1 \quad$ Related Research}

Image texture refers to the local characteristic pattern of image intensity that may be used to identify a tissue. Texture, by definition, also determines local spectral or frequency content in an image; in so far as changes in local texture will cause changes in the local spatial frequency. Aspects of texture in an MR image can thus be quantified by assessing the local spatial frequency content using a space-frequency transform: strong low frequencies appear as homogenous smooth regions, while strong high frequencies are seen as heterogeneous detailed regions.

Texture patterns have been shown to correlate with tissue histopathology in models of multiple sclerosis. In particular, Zhang et al. 6] characterized image texture in vivo using the polar S-Transform (pST) of histologically verified multiple sclerosis lesions within T2-weighted MRI. Both high and low frequency components, representing inflammation and demyelination, were significantly elevated in pathological regions compared to normal control tissue. Their work was one of the first studies to suggest that local spatial-frequency measures of image texture may provide a sensitive and precise indication of disease activity.

Likewise, in [7, researchers applied a variant of the S-transform similar to the pST in order to extract texture features from T2, FLAIR, and T1-postcontrast MR images of patients with oligodendrogliomas, a tumor related to GBM. Their study produced a highly accurate classifier capable detecting the co-deletion status of $1 \mathrm{p}$ and $19 \mathrm{q}$ chromosomes, a favorable genotype associated with slowgrowing oligodendrogliomas. Unfortunately, none of the aforementioned S-Transform based techniques have produced classifiers with high enough accuracy for clinical use in predicting the methylation status of GBM's 8 ]. 
$\mathrm{T} 2$ FLAIR T1-postcontrast tumor ROI
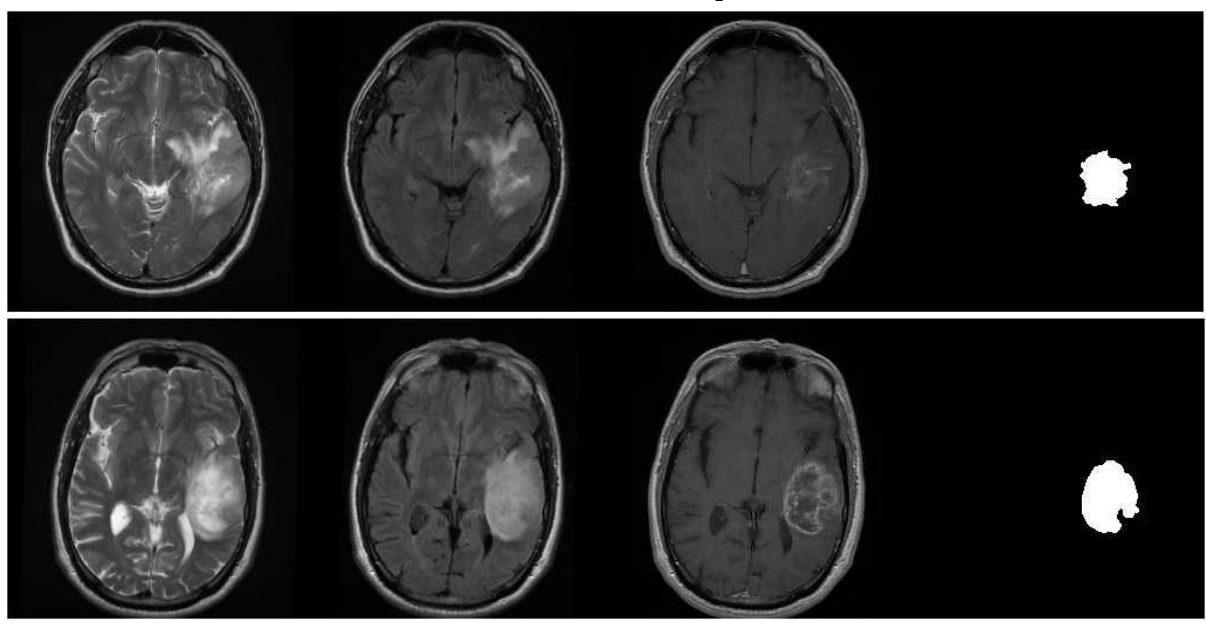

Fig. 1. Methylated (Top) versus Unmethylated (Bottom) tumor appearance. Leftto-Right: T2 image, FLAIR image, T1-postcontrast image, tumor ROI. The most prominent feature of a methylated tumor is the diffuse border visible in all three MRI modalities. In addition, unmethylated tumors tend to exhibit more extensive necrosis and are more likely to appear as ring enhancing within the T1-postcontrast image.

\section{Texture Analysis and Classification}

\subsection{D-DOST Feature Extraction}

To quantify image texture within an ROI, the 2-dimensional variant discrete orthogonal S-transform (2D-DOST) was utilized. Recent results by Drabycz et. al. [5], indicates that the rotationally invariant DOST outperforms leading waveletbased texture analysis methods. The spatial-frequency technique extracts texture features by decomposing an MR image into a set of images at various spatial frequencies. With 2D-DOST a local spatial frequency spectrum describing the amplitude of each frequency component in cycles per $\mathrm{cm}\left(\mathrm{cm}^{-1}\right)$ from the lowest (the average of the entire image) to the highest (the fluctuations between neighboring pixels) is obtained for each pixel in the original image. The number of points in the spectrum is proportional to the image or ROI size.

Formally, let $(i, j)$ index a discrete set of sites on a spatially regular $N \times M$ lattice:

$$
S=\{(i, j) \mid 1 \leq i \leq N, 1 \leq j \leq M\}
$$

The discrete 2-D Fourier transform (2D-FT) of a function $g(i, j)$ and its inverse, defined on lattice $S$ are given by:

$$
G(u, v)=\sum_{i=0}^{N-1} \sum_{j=0}^{M-1} g(i, j) e^{-(\sqrt{-1}) 2 \pi\left(\frac{u i}{N}+\frac{v j}{M}\right)}
$$




$$
g(i, j)=\frac{1}{N M} \sum_{u=0}^{N-1} \sum_{v=0}^{M-1} G(u, v) e^{(\sqrt{-1}) 2 \pi\left(\frac{u i}{N}+\frac{v j}{M}\right)}
$$

The 2D-DOST of a $N \times N$ image $g$ is calculated by partitioning the 2D-FT of the image, $G$, multiplying by the square root of the number of points in the partition, and performing an inverse 2D-FT. For given frequency orders $p_{i}, p_{j}>1$ we extract the part of the Fourier spectrum where $m=2^{p_{i}-1}$ to $2^{p_{i}}-1$ and $n=2^{p_{j}-1}$ to $2^{p_{j}}-1$ and perform a circular shift by half of the bandwidth:

$$
G_{p_{i}, p_{j}}\left[\frac{m}{N}, \frac{n}{N}\right]=\left\{\begin{array}{rlrl}
G\left[\frac{m+2^{p_{i}}}{N}, \frac{n+2^{p_{j}}}{N}\right], & \text { for } m & =\left[-2^{p_{i}-2},-1\right] \\
G\left[\frac{m+2^{p_{i}-1}}{N}, \frac{n+2^{p_{j}-1}}{N}\right], & \text { for } m & =\left[-2^{p_{j}-2},-1\right] \\
n & =\left[0,2^{p_{i}-2}\right] \\
n & =\left[0,2^{p_{j}-2}\right]
\end{array}\right.
$$

The 2D-DOST is then calculated by taking the 2D-FT of each scaled, shifted part of the Fourier spectrum:

$$
D_{p_{i}, p_{j}}\left[i^{\prime}, j^{\prime}\right]=\frac{1}{\sqrt{2^{p_{i}+p_{j}-2}}} \times \sum_{m=-2^{p_{i}-2}}^{2^{p_{i}-2}-1} \sum_{n=-2^{p_{j}-2}}^{2^{p_{j}-2}-1} G_{p_{i}, p_{j}}\left[\frac{m}{N}, \frac{n}{N}\right] e^{2 \pi\left(\frac{m i^{\prime}}{2^{p_{i}-1}}+\frac{n j^{\prime}}{2^{p_{j}}}\right)}
$$

Based on the 2D-DOST, a rotationally invariant spectrum, was created by averaging specific frequency orders together [5. The process is depicted in Figure 2. The average spectrum from all pixels within the tumor volume was then calculated to obtain a single spectrum for each patient; pixels not within the tumor masks were excluded from analysis. Furthermore, slices where the visible tumor area was less than $50 \mathrm{~mm}^{2}$ were excluded from analysis as well. To remove edge effects and other artifacts that might interfere with the analysis, the aforementioned texture extraction process was carried out on $16 \times 16$ pixel ROIs extracted from the binary mask of each tumor slice. Finally, the spectra were log-transformed and z-scaled (to zero mean and standard deviation of one) prior to application of neural networks in order to stabilize the variance.

\subsection{Artificial Neural Networks}

The standard 2-layer neural network [9] is defined by:

$$
\left.h_{\boldsymbol{\omega}}(\boldsymbol{x})=\boldsymbol{W}_{2} \tanh \left(\boldsymbol{W}_{1} \boldsymbol{x}+\boldsymbol{b}_{1}\right)+\boldsymbol{b}_{2}\right)
$$

where $\boldsymbol{\omega}=\left\{\boldsymbol{W}_{1}, \boldsymbol{W}_{2}, \boldsymbol{b}_{1}, \boldsymbol{b}_{2}\right\}$ are the parameters to be learned based on a set of training pairs $\left\langle\boldsymbol{x}_{k}, \boldsymbol{y}_{k}\right\rangle_{k=1}^{n}$ with input vector $\boldsymbol{x} \in \mathbb{R}^{d}$ and $\boldsymbol{y}$ corresponding to the target output. In our case, $\boldsymbol{x}$ is the set of MRI texture coefficients extracted by the 2D-DOST and $y \in\{+1,-1\}$ indicates the methylation status of a given subject. The matrices $\boldsymbol{W}_{1}, \boldsymbol{W}_{2}$ connect the input layer to the hidden layer, and the hidden layer to the output layer (respectively). To prevent overfitting $\ell_{1}$ regularization 10 is employed in conjunction with error minimization as follows:

$$
E\left(\boldsymbol{y}, \boldsymbol{h}_{\boldsymbol{\omega}}(\boldsymbol{x}), \lambda\right)=\left\|\boldsymbol{y}-\boldsymbol{h}_{\boldsymbol{\omega}}(\boldsymbol{x})\right\|_{\ell_{2}}-\lambda\|\boldsymbol{\omega}\|_{\ell_{1}}
$$




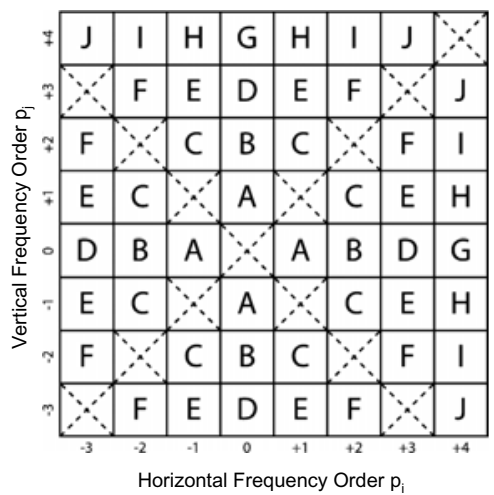

Fig. 2. Rotationally invariant features generated from 2D-DOST $(N=8)$. Features marked with the same letter are averaged together to get a rotation invariant spectrum. The diagonal elements, where $\left|p_{i}\right|=\left|p_{j}\right|$, are excluded, since they tend to contain the majority of the noise and thus degrade classification performance.

where the $\ell_{p}$-norm for vector $\boldsymbol{x}$ is defined as:

$$
\|\boldsymbol{x}\|_{\ell_{p}}=\left(\sum_{i=1}^{d}|x(i)|^{p}\right)^{\frac{1}{p}}
$$

The stochastic weight updates are then defined by:

$$
\begin{aligned}
\boldsymbol{\delta}_{j} & =\boldsymbol{W}_{j}-\eta \frac{\partial E_{m s e}\left(\boldsymbol{y}, \boldsymbol{h}_{\boldsymbol{\omega}}(\boldsymbol{x})\right)}{\partial \boldsymbol{W}_{j}} \\
\boldsymbol{W}_{j} & =\operatorname{sign}\left(\boldsymbol{\delta}_{j}\right) \max \left(0, \boldsymbol{\delta}_{j}-\eta \lambda\right)
\end{aligned}
$$

where $j \in\{1,2\}, \frac{\partial E_{m s e}}{\partial \boldsymbol{W}_{j}}$ is the error gradient, $\eta$ is the learning rate, $\lambda$ is the regularization parameter, and the function $\operatorname{sign}(\boldsymbol{A})$ returns \pm 1 based on the sign of each matrix element in $\boldsymbol{A}$.

From a Bayesian point of view[10], $\ell_{1}$-regularization induces a Laplacian prior over the weights. In contrast to weight decay (i.e., $\ell_{2}$-regularization), $\ell_{1}$ regularization, can drive the weights completely to zero, rather than simply make their magnitudes small and thus enables feature selection. If the $\ell_{1}$-norm of $j^{\text {th }}$ column vector is zero (i.e., $\|\boldsymbol{W}(\cdot, j)\|_{\ell_{1}}=0$ ), then the $j^{\text {th }}$ feature, $\boldsymbol{x}(j)$, is never used and can therefore be removed. Analogously, the width of the hidden layer can be controlled by letting $\ell_{1}$-regularization prune unnecessary hidden nodes. Once again, if a given column vector is zero within weight matrix $\boldsymbol{W}_{2}$, the corresponding hidden unit is effectively ignored by the output layer.

\section{Experimental Procedure}

Patients with newly diagnosed GBM (astrocytoma grade IV, WHO classification) were identified and included in the study based on: (i) age (18 years or 
older), (ii) existence of preoperative T2, FLAIR and T1-post contrast MR images, and (iii) existence of paraffin embedded GBM tissue from the first surgery enabling the assessment of MGMT promoter status via MS-PCR [3].

\subsection{MRI Signal Preprocessing}

Because imaging parameters varied across the cohort, all images were re-sampled to ensure a common field-of-view (FOV) and pixel resolution. Images were cropped and/or zero-padded to achieve a $22 \mathrm{~cm}$ FOV. The $2 \mathrm{D}$ Fourier transform of each image was cropped and/or zero-padded to achieve a consistent image resolution of $0.859 \mathrm{~mm} /$ pixel. The resulting processed images had a FOV=22cm and matrix size of 256x256. A rigid registration for all MR sequences on each case was performed by maximizing the normalized mutual information metric using in-house software. Each volume was converted from 16-bit integer to floating point values and normalized such that cerebrospinal fluid (CSF) in the anterior horn of the left ventricle (or right ventricle if the left was obscured) had an average value of: 1.0 for FLAIR, 5.0 for T2, and 2.0 for T1 post-contrast with a standard deviation of 0.1 . Tumor boundaries, outlined on T1 post-contrast images using MIPAV [11, were utilized for creating the regions of interest (ROIs) demarcating the tumor regions. Figure 1 presents examples of the collected data.

Fifty-nine patients (39 men; 20 women) were included in the texture study, with median age 59 years (range 29-82) at the time of diagnosis. Thirty-one subjects had tumors that were methylated (53\%). Median imaging parameters were as follows for T2, FLAIR and T1-post contrast: TR=4160/9004/500 ms, $\mathrm{TE}=102 / 105 / 14 \mathrm{~ms}, 19$ slices; median inversion time for FLAIR $=2400 \mathrm{~ms}$.

For each T2, FLAIR, and T1-postcontrast imaging modality, 10 rotationally invariant texture feature coefficients were computed using the 2D-DOST 1 The 30 features, after log-normalization and z-scaling, were presented to a neural network in a leave-one-out cross validation strategy (LOOCV). For all experiments the following parameters were kept constant: (i) Learning rate $\eta=0.0005$, number of stochastic weight updates $=10000$, (iii) number of hidden units $=2$ (Note that preliminary experiments varied the number of hidden nodes. However, $\ell_{1}$-regularization consistently pruned the network down to two nodes). The neural network's ability to predict GBM methylation status was evaluated using the following performance indicators: accuracy, sensitivity, specificity, positive, and negative predictive values. Two experiments were performed in this study. The first examined the effect of regularization on the network performance. The second experiment used regularization parameters corresponding to local maxima with respect to accuracy, in order to examine network stability. In each of the 10 trials, the learning parameters ( $\boldsymbol{\omega}$ from Equation 6) were randomly initialized prior to network training and subsequent texture feature classification.

\footnotetext{
${ }^{1}$ Since the Z-dimension of each voxel in our data was approximately 10 times the in-plane dimensions, the relatively straightforward generalization of 2D-DOST to 3 dimensions was not utilized.
} 
Table 1. Neural network prediction as a function of the L1-regularization parameter, $\lambda$ in Equation 10

\begin{tabular}{|c|c|c|c|c|c|}
\hline L1-Regularization & Accuracy & Sensitivity & Specificity & PPV & NPV \\
\hline 0.0001 & 0.898 & 0.893 & 0.903 & 0.893 & 0.903 \\
$\mathbf{0 . 0 0 0 5}$ & $\mathbf{0 . 9 3 2}$ & $\mathbf{0 . 9 2 9}$ & $\mathbf{0 . 9 3 6}$ & $\mathbf{0 . 9 2 9}$ & $\mathbf{0 . 9 3 6}$ \\
0.001 & 0.915 & 0.897 & 0.933 & 0.929 & 0.903 \\
0.005 & 0.898 & 0.867 & 0.931 & 0.929 & 0.871 \\
0.01 & 0.898 & 0.867 & 0.931 & 0.929 & 0.871 \\
$\mathbf{0 . 1}$ & $\mathbf{0 . 9 1 5}$ & $\mathbf{0 . 8 9 7}$ & $\mathbf{0 . 9 3 3}$ & $\mathbf{0 . 9 2 9}$ & $\mathbf{0 . 9 0 3}$ \\
0.2 & 0.848 & 0.828 & 0.867 & 0.857 & 0.839 \\
\hline
\end{tabular}

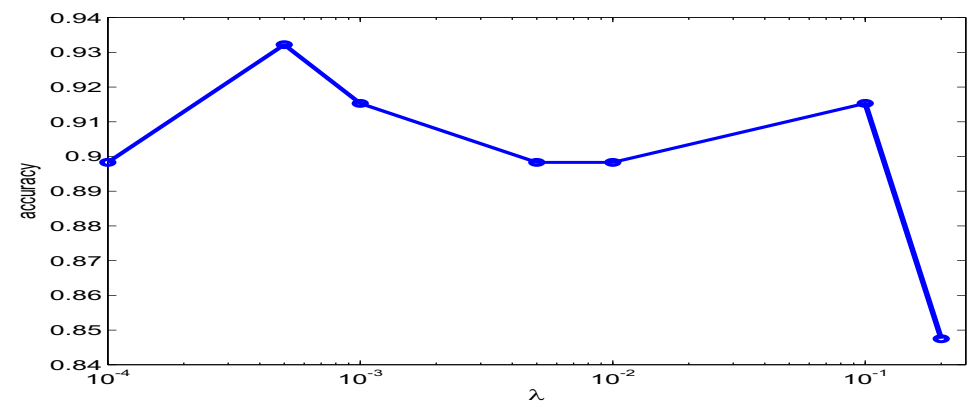

Fig. 3. Accuracy as a function of $\ell_{1}$-Regularization parameter $\lambda$ from Equation 10 . Data taken from Table 1

Table 2. Performance based on 10 random initializations of the network parameters. Top: Performance based on parameter $\lambda=0.1$ in Equation 10 Bottom: Performance based on $\lambda=0.0005$.

\begin{tabular}{|c|c|c|c|c|c|}
\hline $\mathrm{L} 1=0.1$ & Accuracy & Sensitivity & Specificity & PPV & NPV \\
\hline Best Run & 0.915 & 0.897 & 0.933 & 0.929 & 0.903 \\
\hline Worst Run & 0.814 & 0.815 & 0.813 & 0.786 & 0.839 \\
\hline Mean & 0.877 & 0.854 & 0.900 & 0.893 & 0.862 \\
\hline Standard deviation & 0.042 & 0.039 & 0.047 & 0.053 & 0.036 \\
\hline L1 $=0.0005$ & Accuracy & Sensitivity & Specificity & PPV & NPV \\
\hline Best Run & 0.932 & 0.962 & 0.909 & 0.893 & 0.968 \\
\hline Worst Run & 0.661 & 0.633 & 0.690 & 0.679 & 0.645 \\
\hline Mean & 0.812 & 0.814 & 0.812 & 0.789 & 0.833 \\
\hline Standard deviation & 0.071 & 0.089 & 0.058 & 0.059 & 0.088 \\
\hline
\end{tabular}

\subsection{Results}

Table 1 shows the effect of regularization on network performance. Two local maxima exist at $\lambda=\{0.0005,0.1\}$ with respective accuracy of $93.2 \%$ and $91.5 \%$ which can be clearly observed in Figure 3 . In turn, Table2 shows the average performance from 10 random initialization trials performed with the regularization parameters set to $\lambda=\{0.0005,0.1\}$. Performance based on $\lambda=0.1$ indicates this regularization setting is a more stable solution that achieves higher average 
score across all metrics, while also attaining a lower standard deviation than the $\lambda=0.0005$ setting. With $\lambda=0.1$, the average accuracy was $87.7 \%$. The worstcase accuracy remained above $80 \%$, an important threshold for clinical utility. The best-case accuracy was on par with that from physical biopsy $(\sim 90 \%)$.

\section{Discussion}

In this study, we sought to identify a quantitative texture pattern in MR images that is significantly associated with MGMT promoter methylation status. We hypothesized that textural features would correlate with MGMT status, providing a non-invasive imaging test for detection of MGMT promoter methylation in GBM. Using the 2D-DOST in conjunction with neural networks we were able to create a system for accurately predicting methylation status of a given GBM. Our system achieved an average accuracy of $87.7 \%$. The worst-case accuracy remained above $80 \%$, an important threshold for clinical utility. The best-case accuracy was on par with that from physical biopsy $(\sim 90 \%)$. We therefore conclude that our proposed virtual biopsy technique may complement traditional biopsies, particularly for patients in whom direct testing is inconclusive, or infeasible.

\section{References}

1. Stupp, R., Mason, W., van den Bent, M., Weller, M., Fisher, B., Taphoorn, M., Belanger, K., Brandes, A., Bogdahn, C.M.U., Curschmann, J., Janzer, R., Ludwin, S., Gorlia, T., Allgeier, A., Lacombe, D., Cairncross, J., Eisenhauer, E., Mirimanoff, R.: Radiotherapy plus concomitant and adjuvant temozolomide for glioblastoma. New England Journal of Medicine 352(10), 987-996 (2005)

2. Peter, H., Roger, S.: MGMT methylation status: the advent of stratified therapy in glioblastoma? Disease markers 23(1-2), 97-104 (2007)

3. Hegi, M., Diserens, A., Gorlia, T., Hamou, M., de Tribolet, N., et al.: MGMT gene silencing and benefit form temozolomide in glioblastoma. New England Journal of Medicine 352(10), 997-1003 (2005)

4. Eoli, M., Menghi, F., Bruzzone, M., Simone, T.D., Valletta, L., Pollo, B., Bissola, L., Silvani, A., Bianchessi, D., D'Incerti, L., Filippini, G., Broggi, G., Boiardi, A., Finocchiaro, G.: Methylation of o6-methylguanine dna methyltransferase and loss of heterozygosity on $19 q$ and/or $17 p$ are overlapping features of secondary glioblastomas with prolonged survival. Clinical Cancer Research 13(9), 2606-2613 (2007)

5. Drabycz, S., Stockwell, R.G., Mitchell, R.: Image texture characterization using the discrete orthonormal s-transform. Journal of Digital Imaging (2008), doi:10.1007/s10278-008-9138-8

6. Zhang, Y., Wells, J., Buist, R., Peeling, J., Yong, V.W., Mitchell, J.R.: A novel MRI texture analysis of demyelination and inflammation in relapsing-remitting experimental allergic encephalomyelitis. In: Larsen, R., Nielsen, M., Sporring, J. (eds.) MICCAI 2006. LNCS, vol. 4190, pp. 760-767. Springer, Heidelberg (2006)

7. Brown, R., Zlatescu, M., Sijben, A., Roldan, G., Easaw, J., Forsyth, P., Parney, I., Sevick, R., Yan, E., Demetrick, D., Schiff, D., Cairncross, G., Mitchell, R.: The use of magnetic resonance imaging to noninvasively detect genetic signatures in oligodendroglioma. Clinical Cancer Research 14, 2357-2362 (2008) 
8. Drabycz, S.: Effcient S-Transform Techniques for Magnetic Resonance Imaging. PhD thesis, University of Calgary (2009)

9. Haykin, S.: Neural Networks: A Comprehensive Foundation. Macmillian College Pub. Co. (1994)

10. Williams, P.M.: Bayesian regularisation and pruning using a laplace prior. Neural Computation 7, 117-143 (1995)

11. McAuliffe, M.J., Lalonde, F.M., McGarry, D., Gandler, W., Csaky, K., Trus, B.L.: Medical image processing, analysis \& visualization in clinical research. In: CBMS 2001: Proceedings of the Fourteenth IEEE Symposium on Computer-Based Medical Systems, Washington, DC, USA, p. 381. IEEE Computer Society, Los Alamitos (2001) 